\title{
Stage IA Mycosis Fungoides AJCC v7
}

National Cancer Institute

\section{Source}

National Cancer Institute. Stage IA Mycosis Fungoides A/CC v7. NCI Thesaurus. Code C130951.

Stage IA includes: T1, N0, M0, B0-1. T1: Limited patches, papules, and/or plaques covering less than $10 \%$ of the skin surface. May further stratify into T1a (patch only) vs. T1b (plaque +/- patch). N0: No clinically abnormal peripheral lymph nodes; biopsy not required. M0: No visceral organ involvement. B0: Absence of significant blood involvement: $5 \%$ or less of peripheral blood lymphocytes are atypical (Sezary cells). B1: Low blood tumor burden: more than $5 \%$ of peripheral blood lymphocytes are atypical (Sezary cells) but does not meet the criteria of B2. (AJCC 7th ed.) 